\title{
Hyperspectral remote sensing for light pollution monitoring
}

\author{
Alessandro Barducci $\left({ }^{1}\right)$, Marco Benvenuti $\left({ }^{2}\right)\left({ }^{3}\right)$, Laura Bonora $\left({ }^{2}\right)\left(\left(^{3}\right)\right.$, Francesco Castagnoli $\left({ }^{1}\right)$, \\ Donatella Guzzi $\left({ }^{1}\right)$, Paolo Marcoionni $\left({ }^{1}\right)$ and Ivan Pippi $\left({ }^{1}\right)$ \\ $\left.{ }^{1}{ }^{(}\right)$Istituto di Fisica Applicata «Nello Carrara» (IFAC), CNR, Firenze, Italy \\ $\left.{ }^{2}{ }^{2}\right)$ Istituto di Biometeorologia (IBIMET), CNR, Firenze, Italy \\ $\left.{ }^{3}\right)$ Centro di Studi per l'Applicazione dell'Informatica in Agricoltura (CeSIA), \\ Accademia dei Georgofili, Firenze, Italy
}

\begin{abstract}
A possible application of hyperspectral remote sensing regards the assessment of light pollution due to cities and industries. In this paper we introduce the results from a remote sensing campaign performed in September 2001 at night time. For the first time nocturnal light pollution was measured at high spatial and spectral resolution using two airborne hyperspectral sensors, namely the Multispectral Infrared and Visible Imaging Spectrometer (MIVIS) and the Visible InfraRed Scanner (VIRS-200). These imagers, generally employed for day-time Earth remote sensing, were flown over the Tuscany coast (Italy) on board of a Casa 212/200 airplane from an altitude of 1.5-2.0 km. We describe the experimental activities which preceded the remote sensing campaign, the optimization of sensor configuration, and the images as far acquired. The obtained results point out the novelty of the performed measurements and highlight the need to employ advanced remote sensing techniques as a spectroscopic tool for light pollution monitoring.
\end{abstract}

Key words light pollution monitoring - line emission - hyperspectral remote sensing - airborne imaging spectrometers - data processing

\section{Introduction}

Light pollution, a problem that affects almost any urban areas, is produced by a large number of lighting sources, which spill light into the sky. Due to the presence of dust and aerosols in the atmosphere the light is scattered, brightening the sky (Catanzaro and Catalano, 2000; Cinzano et al., 2001). One of the effects

Mailing address: Dr. Ivan Pippi, Istituto di Fisica Applicata «Nello Carrara» (IFAC), CNR, Via Madonna del Piano 10, 50019 Sesto Fiorentino (Firenze), Italy; e-mail: I.Pippi@ifac.cnr.it of the brightened sky is that stars and other astronomical objects, that are relatively faint, are lost in the background glow.

Many studies have been performed to measure the effects of light pollution directly or indirectly (Garstang, 1989, 1991, 2000). In-field measurements have high accuracy but do not have the spatial extension necessary to compute light pollution over large areas.

After the digital Operational Line Scan (OLS) instruments began their services on board of the NOAA-Defense Meteorological Satellite Program (DMSP), the remote assessment of light pollution gained new possibilities, complementing the information derived from groundbased measurements (Isobe, 1998; Isobe and Kosai, 1998; Isobe and Hamamura, 1998; Walker, 1973). The main properties of the DMSP-OLS instrument are shown in table I. However, satellite measurements as far performed do not provide the user with information about the spec- 
Table I. Main properties of the DSMP-OLS instrument used for the remote assessment of light pollution.

\begin{tabular}{ll}
\hline \hline Type & OLS - Oscillating Scan Radiometer \\
Sensor & Photo Multiplier Tube (PMT) \\
Satellite & NOAA-DMSP, sun-synchronous polar orbit \\
Altitude and orbital period & $830 \mathrm{~km}, 101 \mathrm{~min}$ \\
Spectral range & $0.41-0.99 \mu \mathrm{m}$ \\
& $10.0-13.4 \mu \mathrm{m}$ \\
Spatial resolution & $2.8 \mathrm{~km}$ at nadir (on-board averaging of $5 \times 5$ blocks at $0.56 \mathrm{~km}$ ) \\
Swath & $3000 \mathrm{~km}$ \\
Dynamic range & $10^{-9} \mathrm{~W} \mathrm{~cm}^{-2} \mathrm{sr}^{-1} \mu \mathrm{m}^{-1}$ \\
\hline
\end{tabular}

trum of the observed source, and this circumstance originates additional difficulties for modeling atmospheric effects.

In order to investigate the application of standard hyperspectral remote sensing imagers to light pollution monitoring a nocturne aerial survey was performed over the Tuscany coast (Italy) in September 2001.

In Section 2 we present the measurements of nocturnal light pollution obtained for the first time at high spatial and spectral resolution using the Multispectral Infrared and Visible Imaging Spectrometer (MIVIS) and the Visible InfraRed Scanner (VIRS 200). Section 3 is devoted to the analysis of the gathered hyperspectral data and to the discussion of the obtained results. Finally, Section 4 draws some conclusions.

\section{The remote sensing campaign}

MIVIS is an imaging spectrometer, which operates in whisk-broom mode and collects image data from 102 independent spectral channels covering the visible, the near and medium infrared up to $2500 \mathrm{~nm}$, and the thermal infrared spectral range with 10 channels. The extended spectral coverage together with the fine radiometric accuracy (12 bits per radiance sample), makes it an ideal sensor for many remote sensing applications.

VIRS-200 is a push-broom imaging spectrometer operating in the visible and near infrared spectral range. The sensor acquires 20 out of the 240 available spectral channels in the range of 400-1000 $\mathrm{nm}$ with a constant wavelength step of $2.5 \mathrm{~nm}$. The wavelength position of the 20 recorded channels, which are digitised with 10 bits of accuracy, are freely chosen by the operator before the measurement campaign.

In order to map the principal emission lines of common city lights employed for public illumination an optimal spectral configuration was selected. Particularly, four spectral channels were chosen for the observation of the wide spectral emissions from high pressure $\mathrm{Na}$ sources (broad features roughly located near to $570 \mathrm{~nm}$ and $600 \mathrm{~nm}$ ).

Six channels were devoted to the measurement of three $\mathrm{Hg}$ emission lines (at $435.85 \mathrm{~nm}$, $546.07 \mathrm{~nm}$, and the unresolved doublet 576.96 $\mathrm{nm}-579.07 \mathrm{~nm})$. Other six spectral channels were selected for the monitoring of three emission lines from mixed vapour sources, having wavelengths around $450 \mathrm{~nm}, 533 \mathrm{~nm}$ and $586 \mathrm{~nm}$.

The remaining four spectral channels were used as reference and tuned at the following wavelength positions: $506.25 \mathrm{~nm}, 701.25 \mathrm{~nm}$, $801.25 \mathrm{~nm}$, and $936.25 \mathrm{~nm}$. These channels could also observe continuum emission from filament, fluorescent, and very high pressure lamps. The channel at $936.25 \mathrm{~nm}$ falls within a strong atmospheric absorption band due to $\mathrm{H}_{2} \mathrm{O}$ vapour. Table II reports the main technical characteristics of the MIVIS and VIRS-200 sensors.

In fig. 1 we show an uncalibrated spectrum of a city lamp acquired by a portable own-made spectro-radiometer which operates in the 0.4$0.9 \mu \mathrm{m}$ range and acquires 256 samples with a spectral resolution of about $7 \mathrm{~nm}$. This in-field 
Table II. Spectral and radiometric characteristics of the MIVIS and VIRS-200 sensors.

\begin{tabular}{|c|c|c|}
\hline Sensor & MIVIS & VIRS-200 \\
\hline Type & Whisk-broom imaging spectrometer & Push-broom imaging spectrometer \\
\hline Number of channels & 102 & 20 selectable from 240 available \\
\hline Spectral range and resolution & $\begin{array}{l}20 \mathrm{ch} \text {. from } 0.43 \text { to } 0.83 \mu \mathrm{m} \\
(20 \mathrm{~nm} \text { FWHM) } \\
8 \mathrm{ch} \text {. from } 1.15 \text { to } 1.55 \mu \mathrm{m} \\
(50 \mathrm{~nm} \text { FWHM) } \\
64 \mathrm{ch} \text {. from } 1.985 \text { to } 2.479 \mu \mathrm{m} \\
(9 \mathrm{~nm} \text { FWHM) } \\
10 \mathrm{ch} \text {. from } 8.21 \text { to } 12.7 \mu \mathrm{m} \\
(360 \mathrm{~nm} \mathrm{FWHM})\end{array}$ & $\begin{array}{l}0.4-1.0 \mu \mathrm{m} \\
(2.5 \mathrm{~nm} \text { FWHM })\end{array}$ \\
\hline $\mathrm{IFoV}$ & $2.0 \mathrm{mrad}$ & $1.0 \mathrm{mrad}$ \\
\hline Spatial sampling interval & $1.64 \mathrm{mrad}$ & $1.33 \mathrm{mrad}$ \\
\hline Cross-track samples & 755 & 512 \\
\hline Scan rate & $6.25-8.3-12.5-16.7-25 \mathrm{scan} / \mathrm{s}$ & $12-20-30 \mathrm{scan} / \mathrm{s}$ \\
\hline Quantisation accuracy & 12 bit & 10 bit \\
\hline SNR (@albedo 0.5) & $200-800$ up to $1.3 \mu \mathrm{m}$ & $20-400$ \\
\hline
\end{tabular}

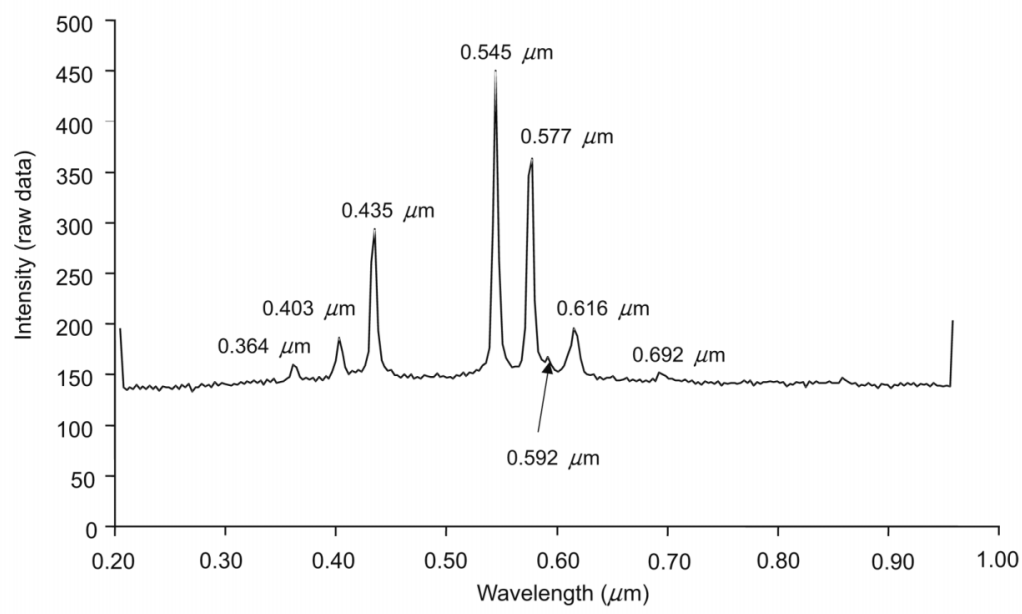

Fig. 1. Uncalibrated spectrum of a standard Hg city lamp emitting white-yellow light. Let us note the fundamental characteristic of this source, which emits radiation in discrete wavelength ranges (spectral lines) associated to transitions between couples of electronic states. Typical mercury spectral emission lines are recognised. Data were acquired by a portable spectro-radiometer developed by us.

instrument was developed at CNR following an original design, and has been described in a previous paper (Barducci et al., 2002).

A CASA 212/200 airplane equipped with the two aforementioned sensors was flown at
$3000 \mathrm{~m}$ of altitude. The flight was performed on September 13, 2001 around 1:00 am GMT, from North-North West toward South-South East, over the Tuscany coast (Italy) from Livorno up to Viareggio. 


\section{Data analysis and discussion}

In order to retrieve spectra of at-sensor radiance VIRS 200 images have been pre-processed

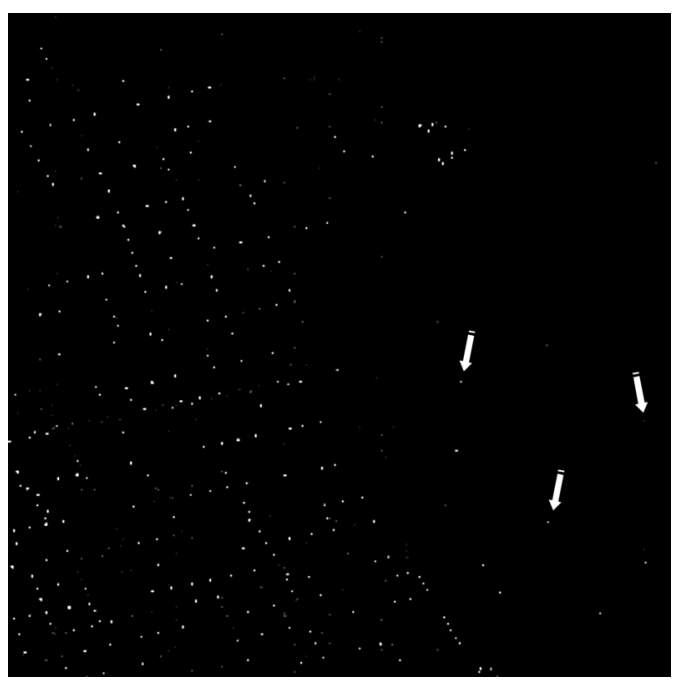

Fig. 2. VIRS 200 grey scale image of Viareggio displayed in the 9th channel $(546.25 \mathrm{~nm})$. White arrows indicate the position of faint city lights. through dark-signal subtraction, flat correction and conversion to radiance unit ( $\mathrm{nW} \mathrm{cm} \mathrm{cr}^{-1}$ $\mathrm{nm}^{-1}$ ) (Barducci and Pippi, 2001). Radiance images were filtered in order to remove noise contributions less than $3 \sigma, \sigma$ being the noise standard deviation.

A VIRS 200 image captured at the 9th channel $(546.25 \mathrm{~nm})$ over Viareggio is displayed in fig. 2. The wavelength position of the channel used for generating this image was selected to map one of the most intense emission lines recognized during the in-field activity and shown in fig. 1. As can be seen, the VIRS 200 imager is able to resolve spectrally and spatially even faint city lights. Spectra of two city lights retrieved from the VIRS 200 data at two different locations are shown in fig. 3. Probably these spectra are recognized as high-pressure $\mathrm{Na}$ lamp. It can be stated that the imaged sources show a maximum scene brightness of the same order of magnitude as that observed during similar day-time measurements.

From analyzing the retrieved spectra, a maximum scene brightness (at-sensor radiance) of the order of $2000-2500 \mathrm{nW} \mathrm{cm}^{-2} \mathrm{sr}^{-1} \mathrm{~nm}^{-1}$ was observed. This amount is near to at-sensor radiance maximum values observed during daytime measurements over low-albedo surface

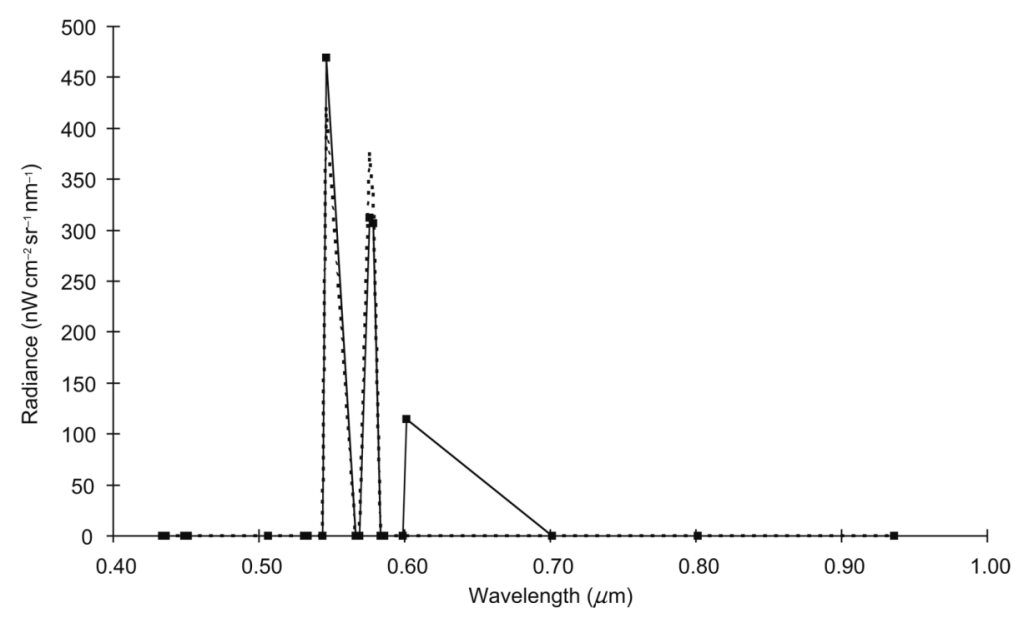

Fig. 3. Spectra of two city lights at two different pixels extracted from the VIRS 200 image, after processing for dark-signal subtraction, flat-field calibration and transformation to radiance units. The square symbols indicate the wavelength position of the VIRS 200 channel. 
like inner-water, asphalt, burned areas, and so forth. These radiance levels could easily be calculated by running, for example, the MODTRAN code.

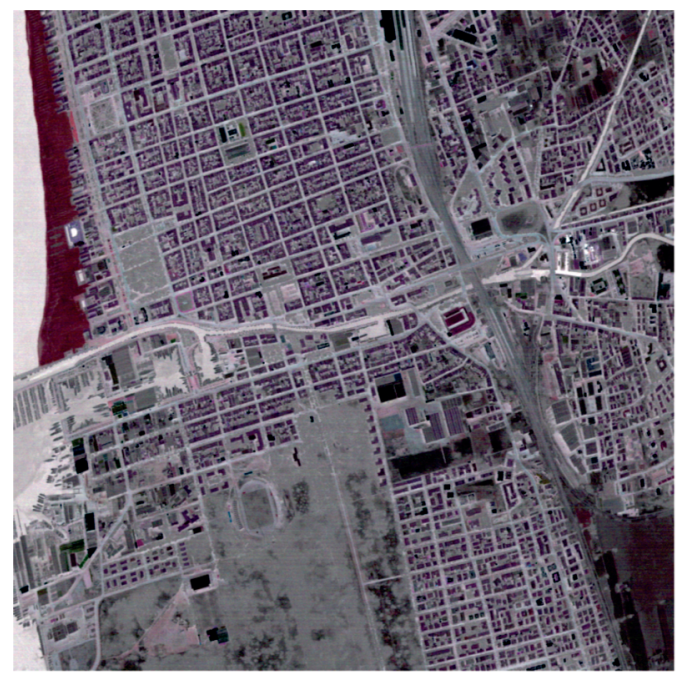

Fig. 4. MIVIS composite image acquired over Viareggio and obtained mapping the 100th channel as red, the 95th channel as green and the 93rd channel as blue.
A MIVIS composite image over Viareggio is displayed in fig. 4 mapping the 100th channel as red, the 95th as green and the 93rd as blue. As expected, the image shows low contrast due to the high intensity correlation degree among thermal infrared wavelengths. We have verified that visible and near infrared MIVIS data are useless for the aim pursued by our research: these spectral channels are not able to detect the faint city lights revealed by the VIRS-200 (MIVIS images at these wavelengths are constituted almost exclusively by noise).

Due to its high sensitivity the VIRS-200 imager detects even faint city lights contrary to what happens with MIVIS sensor. In fact, a narrow spectral channel tuned at a wavelength resonant with a source's emission line senses a higher signal than a broadband channel does. This phenomenon can be explained considering that the instrument measurement in a given channel represents a sort of spectral average of the power emitted by the source, with averaging (integration) extended to the channel's bandwidth. When observing line sources (i.e. sources which emit electromagnetic power concentrated in a sequence of spectral lines rather than as a spectrally continuous power), the resulting spectral average is heavily affected by the channel's band-

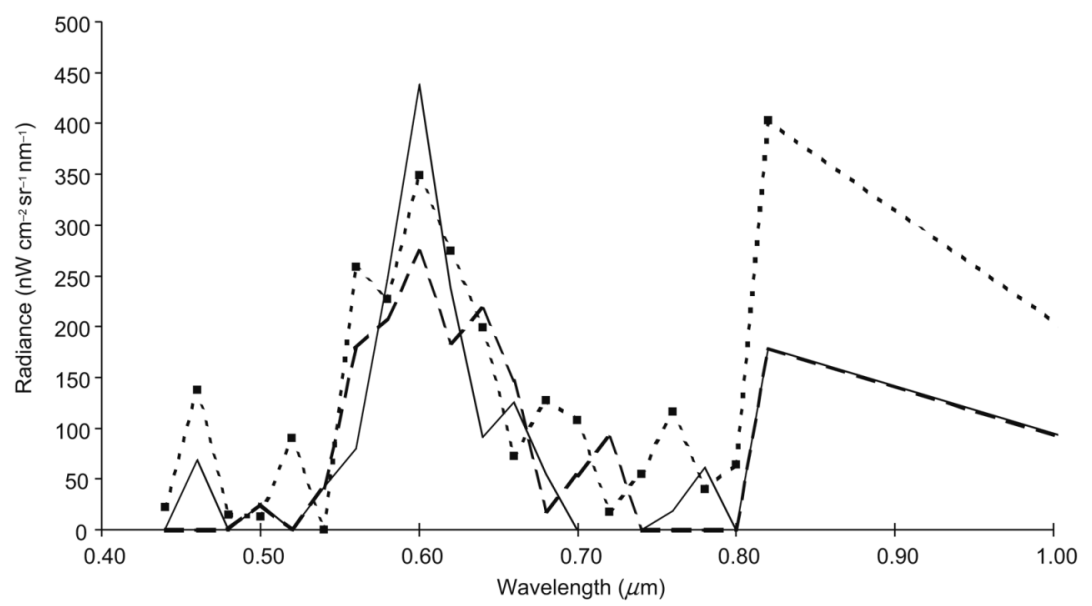

Fig. 5. Visible and near-infrared spectra of three city lights at three different pixels extracted from radiometrically corrected MIVIS image. The square symbols indicate the wavelength position of the MIVIS channel. 
width: if the FWHM of the involved spectral channel largely exceeds the emitted line width, the average will converge to a vanishing value. This is the problem with using MIVIS data for this end, a problem that does not affect VIRS acquisitions that are performed with the finer spectral resolution of $2.5 \mathrm{~nm}$.

In other words, urban lights emit, on average, significantly lower power than solar radiation reflected by soils. However, the difference in emitted radiance between the two sources becomes small in the narrow spectral bands in which city light emission is concentrated.

Let us note that the relative high power emitted by most of the city lights suggests the presence of a certain number of unshielded lights, the radiation of which is directly seen by the sensor. These city lights should be recognized even in the MIVIS images, which instead reveal only a small number of sources. Spectra of three city lights retrieved from the MIVIS data at three different locations are shown in fig. 5.

\section{Conclusions}

In this paper we have investigated the problem of light pollution affecting night sky near urban areas by means of standard remote sensing instruments. A remote sensing campaign has been performed in September 2001 near the Tuscany coast in order to analyze a new approach for the detection of the stray light level.

We have employed two hyperspectral imagers, the MIVIS and the VIRS 200, operating on board of a Casa 212/200 airplane at an altitude between $1.5-3.0 \mathrm{~km}$.

The preliminary results have confirmed that the detection of night sky stray light is possible by means of narrow-band hyperspectral imagers having bandpass channels centered around emission features of common artificial light sources. We have also found that the acquired images show a maximum scene brightness (maximum spectral radiance) that is three to five times less than that observed during day-time acquisitions, provided that measurements are performed with fine enough spectral resolution (not coarser than $3 \mathrm{~nm}$ ). When measurement spectral resolution is significantly worst, the instrument is no longer able to recognize line sources. Hence, even if the emitted power by most of the city lights is relatively high, the MIVIS is only able to detect a small fraction of these sources, which usually show a broadband spectral emission of very high power.

\section{REFERENCES}

BARDUCCI, A. and I. PIPPI (2001): Analysis and rejection of systematic disturbances in hyperspectral remotely sensed images of the Earth, Appl. Opt., 40, 1464-1477.

Barducci, A., P. Marcoionni, I. PiPPI and M. Poggesi (2002): Development of a solar spectro-irradiometer for the validation of remotely sensed hyperspectral images, in Observing our Environment from Space: New Solutions for a New Millennium, edited by G. BEGNI, Proceedings of 21st EARSeL Symposium, 14-16 May 2001, Paris (France), (A.A. Balkema Publishers), 197-202.

Catanzaro, G. and F.A. Catalano (2000): Measurements of the night sky brightness at the Catania astrophysical observatory, Men. S.A.It., 71, 211-220.

Cinzano, P., F. FAlChi and C.D. Elvidge (2001): Nakedeye star visibility and limiting magnitude mapped from DMSP-OLS satellite data, Mon. Not. R. Astron. Soc., 323, 34-46.

GARstanG, R.H. (1989): Night-sky brightness at observatories and sites, Publ. Astron. Soc. Pac., 101, 306-329.

Garstang, R.H. (1991): Dust and light pollution, Publ. Astron. Soc. Pac., 103, 1109-1116.

GARSTANG, R.H. (2000): Light pollution at Mount Wilson: the effects of population growth and air pollution, Men. S.A.It., 71, 71-81.

IsoBE, S. (1998): Bilateral agreements, zoning, international protocol to preserve astronomical windows, Astron. Soc. Pac. Conf. Ser., 139, 119-123.

Isobe, S. and S. Hamamura (1998): Ejected city light of Japan observed by a defense meteorological satellite program, Astron. Soc. Pac. Conf. Ser., 139, 19-199.

Isobe, S. and H. KosAI (1998): Star watching observations to measure night sky brightness, Astron. Soc. Pac. Conf. Ser., 139, 175-184.

WALKER, M.F. (1973): Light pollution in California and Arizona, Publ. Astron. Soc. Pac., 85, 508-519. 